\title{
ON FUNCTIONAL RECORDS AND CHAMPIONS
}

\author{
CLÉMENT DOMBRY, MICHAEL FALK, MAXIMILIAN ZOTT
}

\begin{abstract}
Records among a sequence of iid random variables $X_{1}, X_{2}, \ldots$ on the real line have been investigated extensively over the past decades. A record is defined as a random variable $X_{n}$ such that $X_{n}>\max \left(X_{1}, \ldots, X_{n-1}\right)$. Trying to generalize this concept to the case of random vectors, or even stochastic processes with continuous sample paths, the question arises how to define records in higher dimensions. We introduce two different concepts: A simple record is meant to be a stochastic process (or a random vector) $\boldsymbol{X}_{n}$ that is larger than $\boldsymbol{X}_{1}, \ldots, \boldsymbol{X}_{n-1}$ in at least one component, whereas a complete record has to be larger than its predecessors in all components. The behavior of records is investigated. In particular, the probability that a stochastic process $\boldsymbol{X}_{n}$ is a record as $n$ tends to infinity is studied, assuming that the processes are in the max-domain of attraction of a max-stable process. Furthermore, the distribution of $\boldsymbol{X}_{n}$, given that $\boldsymbol{X}_{n}$ is a record is derived.
\end{abstract}

\section{Introduction and Preliminaries}

Preliminaries. Let $S$ be a compact metric space. A max-stable process (MSP) $\vartheta=\left(\vartheta_{s}\right)_{s \in S}$ is a stochastic process with non-degenerate univariate margins and sample paths in $C(S):=\left\{f \in \mathbb{R}^{S}: f\right.$ continuous $\}$ with the property that there are functions $a_{n} \in C^{+}(S):=\{f \in C(S): f>0\}, b_{n} \in C(S), n \in \mathbb{N}$, such that

$$
\max _{i=1, \ldots, n} \frac{\boldsymbol{\vartheta}^{(i)}-b_{n}}{a_{n}}={ }_{\mathcal{D}} \boldsymbol{\vartheta},
$$

where $\boldsymbol{\vartheta}^{(1)}, \ldots, \boldsymbol{\vartheta}^{(n)}$ are independent and identically distributed (iid) copies of $\boldsymbol{\vartheta}$ and $=_{\mathcal{D}}$ denotes equality in distribution. Note that throughout this paper, each

2010 Mathematics Subject Classification. Primary 60G70.

Key words and phrases. Champions and records $\bullet$ multivariate extreme value distribution $\bullet$ maxstable random vectors $\bullet D$-norm $\bullet$ max-stable processes $\bullet$ max-domain of attraction.

This work was supported by a research grant (VKR023480) for the second author from VILLUM FONDEN. 
operation such as $\max ,<, \geq$, and so on is meant componentwise. The class of max-stable distributions coincides with the class of possible limit distributions of linearly standardized maxima of iid processes, which makes it a class of outstanding interest for extreme value theory. Obviously, the univariate margins of an MSP are max-stable distributions on the real line, and hence belong to the class of either Fréchet, Weibull or Gumbel type of distributions. An MSP $\boldsymbol{\xi}=\left(\xi_{s}\right)_{s \in S}$ in $C(S)$ is commonly called simple max-stable, if each univariate margin is unit Fréchet distributed, i. e. $P\left(\xi_{s} \leq x\right)=\exp \left(-x^{-1}\right), x>0, s \in S$. Different to that, we call an MSP $\boldsymbol{\eta}=\left(\eta_{s}\right)_{s \in S}$ in $C(S) \underline{\text { standard max-stable }}$ (SMSP), if all univariate margins are standard negative exponentially distributed, i. e. $P\left(\eta_{s} \leq x\right)=\exp (x), x \leq 0$, $s \in S$. In that case, $\boldsymbol{\eta}={ }_{d} n \max _{i=1, \ldots, n} \boldsymbol{\eta}^{(i)}$ if $\boldsymbol{\eta}^{(1)}, \ldots, \boldsymbol{\eta}^{(n)}$ are iid copies of $\boldsymbol{\eta}$. It can be shown that a process $\boldsymbol{\vartheta}=\left(\vartheta_{s}\right)_{s \in S}$ with continuous sample paths and univariate margins $G_{s}(x)=P\left(\vartheta_{s} \leq x\right), s \in S, x \in \mathbb{R}$, is an MSP iff $\left(\log \left(G_{s}\left(\vartheta_{s}\right)\right)\right)_{s \in S}$ is an SMSP, see Aulbach et al. (2014).

Denote by $E(S)$ the set of all real valued bounded functions with only finitely many discontinuities and define $\bar{E}^{-}(S):=\{f \in E(S): f \leq 0\}$. We know from Giné et al. (1990) and Aulbach et al. (2013) that a stochastic process $\boldsymbol{\eta}=\left(\eta_{s}\right)_{s \in S}$ is an SMSP iff there exists a stochastic process $\boldsymbol{Z}=\left(Z_{s}\right)_{s \in S}$ with sample paths in $\bar{C}^{+}(S):=\{f \in C(S): f \geq 0\}$ and some constant $c \geq 1$ with $\sup _{s \in s} Z_{s}=c$ almost surely and $E\left(Z_{s}\right)=1, s \in S$, such that

$$
P(\boldsymbol{\eta} \leq f)=\exp \left(-\|f\|_{D}\right):=\exp \left(-E\left(\sup _{s \in S}|f(s)| Z_{s}\right)\right), \quad f \in \bar{E}^{-}(S)
$$

Note that the condition $P\left(\sup _{s \in S} Z_{s}=c\right)=1$ can be weakened to $E\left(\sup _{s \in S} Z_{s}\right)<$ $\infty$, see de Haan and Ferreira (2006).

As a matter of fact, the mapping $\|\cdot\|_{D}$ defines a norm on the linear space $E(S)$. We call it $\underline{D \text {-norm }}$ with generator $\boldsymbol{Z}$. Note that the distribution of a generator is not uniquely determined in general, i. e. there might be several different generators of one $D$-norm, but the condition $\sup _{s \in S} Z_{s}=c$ almost surely yields uniqueness. 
The choice of the function space $E(S)$ instead of $C(S)$ may seem uncommon at first, but in fact it allows the smooth incorporation of the finitedimensional theory on max-stable distributions into the functional setup. By a suitable choice of $f \in E(S)$ we obtain for $s_{1}, \ldots, s_{d} \in S$ and $\boldsymbol{x}=\left(x_{1}, \ldots, x_{d}\right) \leq \mathbf{0}$

$$
P(\boldsymbol{\eta} \leq f)=P\left(\eta_{s_{1}} \leq x_{1}, \ldots, \eta_{s_{d}} \leq x_{d}\right)=\exp \left(-E\left(\max _{i=1, \ldots, d}\left|x_{i}\right| Z_{s_{i}}\right)\right)
$$

where the right-hand side is the de Haan-Resnick-Pickands representation of a multivariate standard max-stable distribution function (df), cf. de Haan and Resnick (1977), Pickands (1981). The mapping $\|\boldsymbol{x}\|_{D}:=E\left(\max _{i=1, \ldots, d}\left|x_{i}\right| Z_{s_{i}}\right), \boldsymbol{x} \in \mathbb{R}^{d}$, defines a multivariate $D$-norm, see Falk et al. (2011) for details.

A stochastic process $\vartheta$ with non-degenerate univariate margins which realizes in $C(S)$ fulfills condition (1) iff there is a continuous process $\boldsymbol{X}$ in the max-domain of attraction of $\vartheta$, i. e. there are some norming functions $c_{n} \in C^{+}(S), d_{n} \in C(S)$, $n \in \mathbb{N}$, such that

$$
\max _{i=1, \ldots, n} \frac{\boldsymbol{X}^{(i)}-d_{n}}{c_{n}} \rightarrow_{\mathcal{D}} \boldsymbol{\vartheta}
$$

where $\boldsymbol{X}^{(1)}, \boldsymbol{X}^{(2)}, \ldots$ are iid copies of $\boldsymbol{X}$ and $\rightarrow_{\mathcal{D}}$ denotes convergence in distribution, that is, weak convergence of the distributions in $\left(C(S),\|\cdot\|_{\infty}\right)$. For details, see e. g. de Haan and Ferreira (2006, Section 9.2). We shortly write $\boldsymbol{X} \in \mathcal{D}(\boldsymbol{\vartheta})$ for (2). Relation (2) implies in particular that $\boldsymbol{X}$ is in the functional domain of attraction of the max-stable process $\boldsymbol{\vartheta}$, that is, for all $f \in E(S)$,

$$
P\left(\max _{i=1, \ldots, n} \frac{\boldsymbol{X}^{(i)}-d_{n}}{c_{n}} \leq f\right)=P\left(\frac{\boldsymbol{X}-d_{n}}{c_{n}} \leq f\right)^{n} \rightarrow_{n \rightarrow \infty} P(\boldsymbol{\vartheta} \leq f) .
$$

For details on the functional domain of attraction, see Aulbach et al. (2013) and Aulbach et al. (2014). Note that in the multivariate context, where random vectors (rv) instead of stochastic processes are considered, (2) and (3) are equivalent. Recall that a max-stable df on $\mathbb{R}^{d}$ is always continuous. 
Outline and terminology. In this paper, we deal with different kinds of records of stochastic processes, generally assuming they are in the max-domain of attraction of an MSP and have continuous univariate marginal df. Let $\boldsymbol{X}, \boldsymbol{X}^{(1)}, \boldsymbol{X}^{(2)}, \ldots$ be an iid sequence of stochastic processes in $C(S)$. We call $\boldsymbol{X}^{(n)}$ a simple record, if $\boldsymbol{X}^{(n)} \not \leq \max _{i=1, \ldots, n-1} \boldsymbol{X}^{(i)}$, and a complete record, if $\boldsymbol{X}^{(n)}>\max _{i=1, \ldots, n-1} \boldsymbol{X}^{(i)}$. We further define

$$
\begin{aligned}
& \underline{\pi}_{n}(\boldsymbol{X}):=P\left(\boldsymbol{X}^{(n)} \text { is a simple record }\right), \\
& \bar{\pi}_{n}(\boldsymbol{X}):=P\left(\boldsymbol{X}^{(n)} \text { is a complete record }\right) .
\end{aligned}
$$

By definition, the first observation $\boldsymbol{X}^{(1)}$ is always a record, so we demand $\underline{\pi}_{1}(\boldsymbol{X})=\bar{\pi}_{1}(\boldsymbol{X})=1$. In the univariate case, where $X, X^{(1)}, X^{(2)}, \ldots$ are simply random variables on the real line, records are much easier to handle, and clearly $\underline{\pi}_{n}(X)=\bar{\pi}_{n}(X)=\frac{1}{n}$. There are many detailed works on univariate record and record times, see Galambos (1987, Sections 6.2 and 6.3) and Arnold et al. (1998). Multivariate records have not been discussed that extensively, yet they haven been approached by e. g. Goldie and Resnick (1989), Goldie and Resnick (1995) or Arnold et al. (1998, Chapter 8).

A concept that is closely related to the field of complete records is the socalled concurrency of extremes, which is due to Dombry et al. (2015). We say that $\boldsymbol{X}^{(1)}, \ldots, \boldsymbol{X}^{(n)}$ are sample concurrent, if

$$
\max _{i=1, \ldots, n} \boldsymbol{X}^{(i)}=\boldsymbol{X}^{(k)} \text { for some } k \in\{1, \ldots, n\}
$$

In that case, we call $\boldsymbol{X}^{(k)}$ the champion among $\boldsymbol{X}^{(1)}, \ldots, \boldsymbol{X}^{(n)}$. We denote the sample concurrence probability by $p_{n}(\boldsymbol{X})$ and obtain due to the iid property

$$
\begin{aligned}
& p_{n}(\boldsymbol{X})=P\left(\bigcup_{i=1}^{n}\left\{\boldsymbol{X}^{(i)}>\max _{1 \leq j \neq i \leq n} \boldsymbol{X}^{(j)}\right\}\right) \\
& =\sum_{i=1}^{n} P\left(\boldsymbol{X}^{(i)}>\max _{1 \leq j \neq i \leq n} \boldsymbol{X}^{(j)}\right)=n P\left(\boldsymbol{X}^{(n)}>\max _{j=1, \ldots, n-1} \boldsymbol{X}^{(j)}\right)=n \bar{\pi}_{n}(\boldsymbol{X}) .
\end{aligned}
$$


Different to records, the concept of multivariate and functional champions is very recent. It has been established in the work of Dombry et al. (2015). In their paper, they derive the limit sample concurrence probability under iid $\operatorname{rv} \boldsymbol{X}^{(1)}, \ldots, \boldsymbol{X}^{(n)}$ in $\mathbb{R}^{d}$. There are also many results on statistical inference in their work.

In Section 3, we generalize the limit sample concurrence probability which has been derived in Dombry et al. (2015, Theorem 2) to the case of stochastic processes with continuous sample paths. Further, we compute the distribution of a champion, given that there actually is one. Section 4 deals with simple record times and the distribution of simple records, where all considerations are restricted to the finitedimensional case.

\section{The DUAL $D$-NORM FUnCTION}

To begin with, we introduce a mapping which is strongly related to the $D$ norm of an SMSP, and which will be important troughout the whole paper. Let $\|\cdot\|_{D}$ be a $D$-norm generated by $\boldsymbol{Z}=\left(Z_{s}\right)_{s \in S}$ in $\bar{C}^{+}(S)$, (recall $E\left(Z_{s}\right)=1$ and $\left.E\left(\sup _{s \in S} Z_{s}\right)<\infty\right)$. We call the mapping

$$
\Downarrow \cdot \Downarrow_{D}: E(S) \rightarrow \mathbb{R}, \quad f \mapsto \Downarrow_{f} \mho_{D}:=E\left(\inf _{s \in S}|f(s)| Z_{s}\right),
$$

the dual $D$-norm function corresponding to $\|\cdot\|_{D}$. Note that, despite the fact that the generator of $\|\cdot\|_{D}$ is not uniquely determined, the dual $D$-norm function $\Downarrow \cdot \Downarrow_{D}$ does not depend on the choice of the generator of $\|\cdot\|_{D}$. This is a consequence of Aulbach et al. (2013, Lemma 6). Therefore, the mapping

$$
\|\cdot\|_{D} \rightarrow \gtrless \cdot u_{D}
$$

is well-defined, although not one-to-one, since different $D$-norms can lead to the same dual $D$-norm function. One can check that the dual $D$-norm function is always zero if there are at least two independent components $\eta_{s}, \eta_{t}$ of the SMSP $\boldsymbol{\eta}$ generated by $\|\cdot\|_{D}$, since Takahashi's theorem (Falk et al. (2011, Theorem 4.4.1)) 
implies that $\eta_{s}, \eta_{t}$ are independent iff $Z_{s}+Z_{t}=\max \left(Z_{s}, Z_{t}\right)$ almost surely, entailing in turn $\min \left(Z_{s}, Z_{t}\right)=0$ almost surely.

Throughout this paper, the following result on SMSP will be crucial. By a copula process, we understand a stochastic process with continuous sample paths such that the univariate margins are uniformly distributed on $(0,1)$. We are interested in copula processes $\boldsymbol{U}$ that are in the max-domain of attraction of an SMSP $\boldsymbol{\eta}$, i. e.

$$
n\left(\max _{i=1, \ldots, n} \boldsymbol{U}^{(i)}-1\right) \rightarrow_{\mathcal{D}} \boldsymbol{\eta}
$$

where $\boldsymbol{U}^{(1)}, \boldsymbol{U}^{(1)}, \ldots$ are iid copies of $\boldsymbol{U}$.

Proposition 2.1. Let $\boldsymbol{U}$ be a copula process with $\boldsymbol{U} \in \mathcal{D}(\boldsymbol{\eta})$ (i. e. (5) holds), where $\boldsymbol{\eta}=\left(\eta_{s}\right)_{s \in S}$ is an SMSP. Let $\|\cdot\|_{D}$ be the D-norm corresponding to $\boldsymbol{\eta}$ and $\boldsymbol{Z}$ be a generator of $\|\cdot\|_{D}$. Then

$$
n(1-P(n(\boldsymbol{U}-1) \leq f)) \rightarrow_{n \rightarrow \infty} E\left(\sup _{s \in S}|f(s)| Z_{s}\right)=\|f\|_{D}, \quad f \in \bar{E}^{-}(S),
$$

and

$$
n P(n(\boldsymbol{U}-1)>f) \rightarrow_{n \rightarrow \infty} E\left(\inf _{s \in S}|f(s)| Z_{s}\right)=\imath f \imath_{D}, \quad f \in \bar{E}^{-}(S) .
$$

REMARK 2.2. We call a stochastic process $\boldsymbol{V}$ with sample paths in $\bar{C}^{-}(S):=\{f \in$ $C(S): f \leq 0\}$ a standard generalized Pareto process (standard GPP), if there is a $D$-norm $\|\cdot\|_{D}$ on $E(S)$ generated by an almost surely bounded generator and some $c>0$ such that

$$
P(\boldsymbol{V} \leq f)=1-\|f\|_{D}
$$

for all $f \in \bar{E}^{-}(S)$ with $\|f\|_{\infty} \leq c$. It can easily be shown that the survival function of $\boldsymbol{V}$ is given by

$$
P(\boldsymbol{V}>t f)=t \imath f \imath_{D}
$$

for $t>0$ close enough to zero. Hence, condition (6) and (7) mean that the upper tail of the distribution of the copula process $\boldsymbol{U}$ is close to that of the shifted standard 
GPP $\boldsymbol{V}+1$. For details on GPP, see e. g. Buishand et al. (2008), Aulbach and Falk (2012a), Aulbach and Falk (2012b), and Ferreira and de Haan (2014).

Proof of Proposition 2.1. Condition (5) implies that $\boldsymbol{U}$ is in the functional domain of attraction of $\boldsymbol{\eta}$, see Aulbach et al. (2013, Proposition 5), i. e.

$$
P(n(\boldsymbol{U}-1) \leq f)^{n} \rightarrow_{n \rightarrow \infty} P(\boldsymbol{\eta} \leq f)=\exp \left(-\|f\|_{D}\right), \quad f \in \bar{E}^{-}(S) .
$$

Now (6) follows from Aulbach et al. (2013, Proposition 8). Next we verify (7). Choose a generator $\boldsymbol{Z}$ of $\|\cdot\|_{D}$ with $P\left(\sup _{s \in S} Z_{s}=c\right)=1$ for some $c \geq 1$. Define a measure $\rho$ on the unit sphere $\bar{C}_{1}^{+}(S):=\left\{g \in \bar{C}^{+}(S):\|g\|_{\infty}=1\right\}$ by

$$
\rho(A):=c P(\boldsymbol{Z} / c \in A), \quad A \subset \bar{C}_{1}^{+}(S) \text { Borel, }
$$

which is the well-known angular measure, see e.g. de Haan and Ferreira (2006, Section 9.4). By transforming to polar coordinates, we identify $\bar{C}^{+}(S)$ with the product space $\bar{C}_{1}^{+}(S) \times(0, \infty)$. For the technical details of this transformation, see de Haan and Ferreira (2006, Section 9.3). On this product space, we define a product measure via $d \nu=d \rho \times d r / r^{2}$. The measure $\nu$ is well-known as the exponent

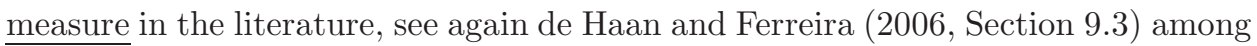
many others. Now having in mind that $\boldsymbol{U}<1$ a.s. (Hofmann (2012, Corollary 3.15)) and $\boldsymbol{\eta}<0$ a.s. (Aulbach et al. (2013, Lemma 1)), it is easy to see that (5) is equivalent with

$$
\frac{1}{n} \max _{i=1, \ldots, n} \frac{1}{1-U^{(i)}} \rightarrow_{\mathcal{D}}-\frac{1}{\eta},
$$

where $-1 / \boldsymbol{\eta}$ is a simple MSP. Therefore, we have

$$
\nu_{n}(A):=n P\left((n(1-\boldsymbol{U}))^{-1} \in A\right) \rightarrow_{n \rightarrow \infty} \nu(A)
$$

for all Borel sets $A \subset \bar{C}^{+}(S)$ with $\nu(\partial A)=0$ and $\inf \left\{\|f\|_{\infty}: f \in A\right\}>0$, see de Haan and Ferreira (2006, Theorem 9.3.1). Define for $h \in E(S)$ the set 
$A_{h}:=\{g \in C(S): g>h\}$. Now, for all $f \in \bar{E}^{-}(S)$ with $f<0$,

$$
\begin{aligned}
n P(n(\boldsymbol{U}-1)>f) & =\nu_{n}\left(A_{-1 / f}\right) \\
& \rightarrow_{n \rightarrow \infty} \nu\left(A_{-1 / f}\right) \\
& =\nu\left(\left\{(g, r) \in \bar{C}_{1}^{+}(S) \times(0, \infty): r g>1 /|f|\right\}\right) \\
& =\int_{\bar{C}_{1}^{+}(S)} \int_{\left(\inf _{s \in S}|f(s)| g(s)\right)^{-1}}^{\infty} r^{-2} \mathrm{~d} r \rho(\mathrm{d} g) \\
& =E\left(\inf _{s \in S}|f(s)| Z_{s}\right) .
\end{aligned}
$$

Remark 2.3. Clearly, the dual $D$-norm function can also be defined for multivariate $D$-norms. Given a multivariate $D$-norm $\|\boldsymbol{x}\|_{D}=E\left(\max _{j=1, \ldots, d}\left|x_{j}\right| Z_{j}\right), \boldsymbol{x} \in \mathbb{R}^{d}$, we write

$$
\text { «x } \Downarrow_{D}:=E\left(\min _{j=1, \ldots, d}\left|x_{j}\right| Z_{j}\right), \quad \boldsymbol{x} \in \mathbb{R}^{d} .
$$

A simple connection between the functions $\|\cdot\|_{D}$ and $\varkappa \cdot \varkappa_{D}$ is now given by the general equation

$$
\min \left(a_{1}, \ldots, a_{d}\right)=\sum_{\emptyset \neq T \subset\{1, \ldots, d\}}(-1)^{|T|-1} \max \left\{a_{j}, j \in T\right\},
$$

which is true for arbitrary numbers $a_{1}, \ldots, a_{d} \in \mathbb{R}$. Applying the inclusion-exclusion principle and including (8), the multivariate version of (7) directly follows from that of (6). While (6) traces back to Deheuvels (1984) and Galambos (1987), the multivariate dual $D$-norm function was established by Schmidt and Stadtmüller (2006), see also de Haan et al. (2008). In their work, $\Downarrow \cdot \Downarrow_{D}$ is called tail copula. However, they do not provide an explicite formula for the tail copula.

EXAMPLE 2.4 (Independence and perfect dependence). We have that

$$
\imath \gtrless_{1}=0
$$


is the least dual $D$-norm function, corresponding to the case of independent univariate margins, where $\|\cdot\|_{D}=\|\cdot\|_{1}$, and

$$
\Downarrow \boldsymbol{x} \Downarrow_{\infty}=\min _{1 \leq j \leq d}\left|x_{j}\right|, \quad \boldsymbol{x} \in \mathbb{R}^{d}
$$

is the largest dual $D$-norm function, corresponding to the perfect dependence case, where $\|\cdot\|_{D}=\|\cdot\|_{\infty}$. Hence, we have for an arbitrary dual $D$-norm function the bounds

$$
0=u_{1} \leq u_{1} \cdot u_{D} \leq u \cdot u_{\infty} .
$$

For the next examples, the following abbreviation is useful. We define for $\boldsymbol{x} \in \mathbb{R}^{d}$ and a nonempty subset $T \subset\{1, \ldots, d\}$

$$
\boldsymbol{x}_{T}:=\left(x_{i}, i \in T\right) \in \mathbb{R}^{|T|} .
$$

ExAmple 2.5 (Fréchet model). It is well-known that a $D$-norm is given by the $l_{\lambda}$-norm

$$
\|\boldsymbol{x}\|_{\lambda}:=\left(\sum_{i=1}^{d}\left|x_{i}\right|^{\lambda}\right)^{1 / \lambda}, \quad \boldsymbol{x} \in \mathbb{R}^{d}, \lambda \in(1, \infty),
$$

usually referred to as the logistic model in the literature. Therefore, we obtain by

$$
\Downarrow \boldsymbol{x} \Downarrow_{\lambda}=\sum_{\emptyset \neq T \subset\{1, \ldots, d\}}(-1)^{|T|-1}\left\|\boldsymbol{x}_{T}\right\|_{\lambda}, \quad \boldsymbol{x} \in \mathbb{R}^{d}, \lambda \in(1, \infty) .
$$

A generator $\boldsymbol{Z}=\left(Z_{1}, \ldots, Z_{d}\right)$ of $\|\cdot\|_{\lambda}$ can easily be found: Put $Z_{i}:=\tilde{Z}_{i} / \Gamma(1-1 / \lambda)$, $i=1, \ldots, d$, where $\tilde{Z}_{1}, \ldots, \tilde{Z}_{d}$ are iid Fréchet distributed with parameter $\lambda$, and $\Gamma$ denotes the gamma function.

Example 2.6 (Weibull model). We can define a generator $\boldsymbol{Z}=\left(Z_{1}, \ldots, Z_{d}\right)$ by taking independent Weibull distributed random variables $\tilde{Z}_{1}, \ldots, \tilde{Z}_{d}$, i. e. $P\left(\tilde{Z}_{1}>\right.$ $t)=\exp \left(-t^{\alpha}\right), t>0, \alpha>0$, and putting $Z_{i}:=\tilde{Z}_{i} / \Gamma(1+1 / \alpha)$. It is easy to show that the corresponding dual $D$-norm function is for $\boldsymbol{x} \in \mathbb{R}^{d}, x_{i} \neq 0, i=1, \ldots, d$, 
given by

$$
\Downarrow \boldsymbol{x}{\Downarrow W_{\alpha}}=\left(\|1 / \boldsymbol{x}\|_{\alpha}\right)^{-1}, \quad \alpha>0 .
$$

Hence, by (8), the attendant $D$-norm is for such $\boldsymbol{x}$

$$
\|\boldsymbol{x}\|_{W_{\alpha}}=\sum_{\emptyset \neq T \subset\{1, \ldots, d\}}(-1)^{|T|-1}\left(\left\|1 / \boldsymbol{x}_{T}\right\|_{\alpha}\right)^{-1}, \quad \alpha>0 .
$$

Note that $\|\cdot\|_{\alpha}$ is defined as in (9), even though it does not define a norm in the case $\alpha<1$.

ExAmple 2.7 (Bernoulli model). A simple example of a discrete generator is induced by independent Bernoulli- $\beta$ random variables $\tilde{Z}_{i}, i=1, \ldots, d, \beta \in(0,1]$, and putting $Z_{i}:=\tilde{Z}_{i} / \beta, i=1, \ldots, d$. The $D$-norm and the dual $D$-norm function are easily derived. We have

$$
\|\boldsymbol{x}\|_{B_{\beta}}=\sum_{\emptyset \neq T \subset\{1, \ldots, d\}} \beta^{|T|-1}(1-\beta)^{d-|T|}\left\|\boldsymbol{x}_{T}\right\|_{\infty}, \quad \boldsymbol{x} \in \mathbb{R}^{d}, \beta \in(0,1] .
$$

Note that $\|\cdot\|_{B_{1}}=\|\cdot\|_{\infty}$ and $\|\cdot\|_{B_{\beta}} \rightarrow\|\cdot\|_{1}$ as $\beta \rightarrow 0$. Analogously,

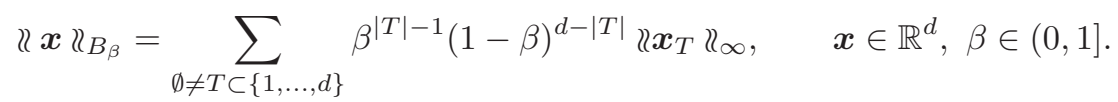

\section{The FunCtional eXtremal CONCURREnCE PRobability}

The aim of this section is to investigate the limit behaviour of the sample concurrence probability. In Dombry et al. (2015), it is shown that the sample concurrence probability $p_{n}(\boldsymbol{X})$ of a $\mathrm{rv} \boldsymbol{X}$ converges, provided that $\boldsymbol{X}$ has continuous margins and lies in the max-domain of attraction of a max-stable rv. We generalize this assertion to the functional setup, having in mind that we can formulate every result in the multivariate context analogously.

Theorem 3.1. Let $\boldsymbol{U}^{(1)}, \boldsymbol{U}^{(2)}, \ldots$ be independent copies of a copula process $\boldsymbol{U}$, satisfying $\boldsymbol{U} \in \mathcal{D}(\boldsymbol{\eta})$, where $\boldsymbol{\eta}$ is an SMSP with corresponding D-norm $\|\cdot\|_{D}$. Then

$$
p_{n}(\boldsymbol{U})=n \bar{\pi}_{n}(\boldsymbol{U}) \rightarrow_{n \rightarrow \infty} E\left(\gtrless \boldsymbol{\eta} \gtrless_{D}\right),
$$


where $\| \cdot \Downarrow_{D}$ is the dual $D$-norm function corresponding to $\|\cdot\|_{D}$.

We call $E\left(\gtrless \boldsymbol{\eta} \gtrless_{D}\right)$ the extremal concurrence probability corresponding to $\|\cdot\|_{D}$, in accordance with the terminology in Dombry et al. (2015). As they have shown, the extremal concurrence probability has the following interpretation. It is wellknown (cf. de Haan and Ferreira (2006, Corollary 9.4.2)), that the simple maxstable process $\boldsymbol{\xi}=-1 / \boldsymbol{\eta}$ has the representation

$$
\boldsymbol{\xi}={ }_{\mathcal{D}} \sup _{k \in \mathbb{N}} \boldsymbol{\vartheta}_{k}
$$

where $\left(\boldsymbol{\vartheta}_{k}\right)_{k \in \mathbb{N}}$ are the points of a Poisson point process on $(0, \infty) \times \bar{C}_{1}^{+}(S)$ with a certain intensity measure. The extremal concurrence probability is now precisely the probability that only one function $\boldsymbol{\vartheta}_{k}$ contributes to the supremum in (11), see Dombry et al. (2015, Theorem 1).

Note that one has to distinguish between $E\left(\Downarrow \boldsymbol{\eta} \gtrless_{D}\right)$ and $E\left(\inf _{s \in S}\left|\eta_{s}\right| Z_{s}\right)$ in general. However, if $\boldsymbol{\eta}$ and $\boldsymbol{Z}$ are independent, both terms coincide, cf. Lemma 3.3 .

Proof of Theorem 3.1. Denote by $P * \xi$ the distribution of a random variable $\xi$. Let $\boldsymbol{\eta}$ be an SMSP with $D$-norm $\|\cdot\|_{D}$ and put $\boldsymbol{M}^{(n)}:=n \max _{i=1, \ldots, n-1}\left(\boldsymbol{U}^{(i)}-1\right) \rightarrow_{\mathcal{D}}$ $\boldsymbol{\eta}$ due to (5). Conditioning on $\boldsymbol{M}^{(n)}=f$ yields

$$
\begin{aligned}
n \bar{\pi}_{n}(\boldsymbol{U}) & =\int_{\bar{C}^{-}(S)} n P(n(\boldsymbol{U}-1)>f)\left(P * \boldsymbol{M}^{(n)}\right)(\mathrm{d} f) \\
& =: \int_{\bar{C}^{-}(S)} G_{n}(f)\left(P * \boldsymbol{M}^{(n)}\right)(\mathrm{d} f)
\end{aligned}
$$

since $\boldsymbol{M}^{(n)}$ and $\boldsymbol{U}$ are independent. Setting $X_{n}:=G_{n} \circ \boldsymbol{M}^{(n)}$, we need to show

$$
n \bar{\pi}_{n}(\boldsymbol{U})=E\left(X_{n}\right) \rightarrow_{n \rightarrow \infty}=E\left(\gtrless \boldsymbol{\eta} \gtrless_{D}\right) .
$$

It is enough to verify (Billingsley (1968, p. 32)):

(i) $X_{n} \rightarrow_{\mathcal{D}} \Downarrow \boldsymbol{\eta} \mho_{D}$.

(ii) There is $\varepsilon>0$ with $\sup _{n \in \mathbb{N}} E\left(\left|X_{n}\right|^{1+\varepsilon}\right)<\infty$. 
Note that (ii) implies the uniform integrability of the sequence $\left(X_{n}\right)_{n \in \mathbb{N}}$.

We first show (i). Obviously, $G_{n}(f) \rightarrow \Downarrow f \Downarrow_{D}$ due to (7). Standard arguments such as the monotone convergence theorem yield $G_{n}\left(f_{n}\right) \rightarrow \mathbb{R} f \mathbb{R}_{D}$ if $f_{n}, f \in \bar{C}^{-}(S)$ with $\left\|f_{n}-f\right\|_{\infty} \rightarrow 0$. Now noticing that $\boldsymbol{M}^{(n)} \rightarrow_{\mathcal{D}} \boldsymbol{\eta}$, the assertion is immediate from the extended continuous mapping theorem, see cf. Billingsley (1968, Theorem $5.5)$.

Now we proof (ii). Elementary calculations show that for all $n \geq 2$

$$
\begin{aligned}
E\left(X_{n}^{2}\right) & =\int_{\bar{C}^{-}(S)} n^{2} P(n(\boldsymbol{U}-1)>f)^{2}\left(P * \boldsymbol{M}^{(n)}\right)(\mathrm{d} f) \\
& \leq \int_{\bar{C}^{-}(S)} n^{2} P\left(n\left(U_{s}-1\right)>f(s)\right)^{2}\left(P * \boldsymbol{M}^{(n)}\right)(\mathrm{d} f) \\
& =E\left(\left(M_{s}^{(n)}\right)^{2}\right)=\frac{2 n}{n+1} \leq 2 .
\end{aligned}
$$

Corollary 3.2. Denote by $M(n):=\sum_{i=1}^{n} 1_{\left\{\boldsymbol{X}^{(i)}>\max _{1 \leq j<i} \boldsymbol{X}^{(j)}\right\}}$ the number of complete records among $\boldsymbol{X}^{(1)}, \ldots, \boldsymbol{X}^{(n)}$. Then

$$
\frac{E(M(n))}{\log (n)} \rightarrow_{n \rightarrow \infty} E\left(\gtrless \boldsymbol{\eta} \Downarrow_{D}\right) .
$$

Proof. The assertion follows from Theorem 3.1 and the fact that $\left(\sum_{i=1}^{n} \frac{a_{i}}{i}\right) / \log (n)$ $\rightarrow_{n \rightarrow \infty} a$, if $\left(a_{n}\right)_{n \in \mathbb{N}}$ is some real-valued sequence with $a_{n} \rightarrow_{n \rightarrow \infty} a$.

The following lemma provides an alternative representation for the extremal concurrence probability. Denote by $1_{A}$ the indicator function of some set $A$, i.e. $1_{A}(\omega)=1$, if $\omega \in A$, and $1_{A}(\omega)=0$, else.

Lemma 3.3. Let $\boldsymbol{\eta}=\left(\eta_{s}\right)_{s \in S}$ be an $S M S P$ in $\bar{C}^{-}(S)$ with $D$-norm $\|\cdot\|_{D}$ and generator $\boldsymbol{Z}=\left(Z_{s}\right)_{s \in S}$, and $f \in \bar{E}^{-}(S)$. Then

$$
E\left(\Downarrow \boldsymbol{\eta} \varkappa_{D}\right)=E\left(\|1 / \boldsymbol{Z}\|_{D}^{-1} 1_{\{\boldsymbol{Z}>0\}}\right) .
$$


(ii)

$$
\begin{aligned}
& E\left(ख \max (\boldsymbol{\eta}, f) \gtrless_{D}\right)= \\
& =E\left(\left(\|1 / \boldsymbol{Z}\|_{D}\right)^{-1}\left(1-\exp \left(\|1 / \boldsymbol{Z}\|_{D} \sup _{s \in S}\left(f(s) Z_{s}\right)\right)\right) 1_{\{\boldsymbol{Z}>0\}}\right) .
\end{aligned}
$$

Proof. Without loss of generality, choose a generator $\boldsymbol{Z}$ of $\|\cdot\|_{D}$ which is independent of $\boldsymbol{\eta}$. Then

$$
E\left(\inf _{s \in S}\left|\eta_{s}\right| Z_{s}\right)=\int_{\bar{C}^{-}(S)} \Downarrow f \Downarrow_{D}(P * \boldsymbol{\eta})(d f)=E\left(\Downarrow \boldsymbol{\eta} \Downarrow_{D}\right) .
$$

Suppose $P(\boldsymbol{Z}>0)=1$ for ease of notation. Fubini's theorem and the fact that $\boldsymbol{\eta}$ and $\boldsymbol{Z}$ are independent, entail

$$
\begin{aligned}
E\left(\inf _{s \in S}\left(\left|\eta_{s}\right| Z_{s}\right)\right) & =\int_{0}^{\infty} P\left(\inf _{s \in S}\left(\left|\eta_{s}\right| Z_{s}\right)>t\right) \mathrm{d} t \\
& =\int_{0}^{\infty} P\left(\eta_{s}<-t / Z_{s}, s \in S\right) \mathrm{d} t \\
& =E\left(\int_{0}^{\infty} \exp \left(-t\|1 / \boldsymbol{Z}\|_{D}\right) \mathrm{d} t\right) \\
& =E\left(\left(\|1 / \boldsymbol{Z}\|_{D}\right)^{-1} \int_{0}^{\infty} \exp (-t) \mathrm{d} t\right),
\end{aligned}
$$

which is (i). Assertion (ii) can be shown by similar arguments.

EXAMPle 3.4 (Independence and perfect dependence). A generator of the special $D$-norm $\|\cdot\|_{D}=\|\cdot\|_{\infty}$, which characterizes the complete dependence of the univariate margins of $\boldsymbol{\eta}$, is obviously given by the constant $\boldsymbol{Z} \equiv 1$. In that case, Theorem 3.1 shows that the extremal concurrence probability is one, i.e. $p_{n}(\boldsymbol{U})=n \bar{\pi}_{n}(\boldsymbol{U}) \rightarrow_{n \rightarrow \infty} 1$. This is not at all surprising: in the univariate context, where $X^{(1)}, \ldots, X^{(n)}$ are random variables on the real line, there clearly exists a champion with probability one - it is the maximum of $X^{(1)}, \ldots, X^{(n)}$.

In contrast to that, we have

$$
E\left(\left(\|1 / \boldsymbol{Z}\|_{D}\right)^{-1} 1_{\{\boldsymbol{Z}>0\}}\right)=0 \Longleftrightarrow \inf _{s \in S} Z_{s}=0 \text { a.s. }
$$


In particular, this is the case when at least two components $\eta_{s}, \eta_{t}, s \neq t$, are independent, see the argument in Section 2.

Example 3.5 (Bernoulli model). Consider a standard max-stable $\mathrm{rv} \boldsymbol{\eta} \in \mathbb{R}^{d}$ with corresponding $D$-norm $\|\cdot\|_{B_{\beta}}, \beta \in(0,1]$, known from Example 2.7. It is easy to see that

$$
\|\mathbf{1}\|_{B_{\beta}}=\frac{1-(1-\beta)^{d}}{\beta}
$$

From the general equality

$$
E\left(\gtrless \tilde{\boldsymbol{\eta}} \mho_{\infty}\right)=\frac{1}{\|\mathbf{1}\|_{D}},
$$

where $\tilde{\boldsymbol{\eta}}$ is some standard max-stable rv with $D$-norm $\|\cdot\|_{D}$, we conclude

$$
\begin{aligned}
E\left(\Downarrow \boldsymbol{\eta} \Downarrow_{B_{\beta}}\right) & =\sum_{\emptyset \neq T \subset\{1, \ldots, d\}} \beta^{|T|-1}(1-\beta)^{d-|T|} E\left(\Downarrow \boldsymbol{\eta}_{T} \Downarrow_{\infty}\right) \\
& =\sum_{k=1}^{d}\left(\begin{array}{l}
d \\
k
\end{array}\right) \beta^{k} \frac{(1-\beta)^{d-k}}{1-(1-\beta)^{k}}
\end{aligned}
$$

For another example, namely the logistic model, we refer to Example 4.4.

REMARK 3.6. (i) Theorem 3.1 implies that the extremal concurrence probability, just like the dual $D$-norm function, does not depend on the choice of $\boldsymbol{Z}$, but only on $\|\cdot\|_{D}$.

(ii) In the preceding theorem, we can replace $\boldsymbol{U}, \boldsymbol{U}^{(1)}, \boldsymbol{U}^{(2)}, \ldots$ by a sequence of iid stochastic processes $\boldsymbol{X}, \boldsymbol{X}^{(1)}, \boldsymbol{X}^{(2)}, \ldots$ whose univariate marginal df $F_{s}(x)=$ $P\left(X_{s} \leq x\right), s \in S$, are continuous and strictly monontonically increasing on their support. The conditions (6) and (7) will then have to apply to the copula process $\left(F_{s}\left(X_{s}\right)\right)_{s \in S}$. In that case,

$$
n P\left(\boldsymbol{X}>\max _{i=1, \ldots, n-1} \boldsymbol{X}^{(i)}\right) \rightarrow_{n \rightarrow \infty} E\left(\left(\|1 / \boldsymbol{Z}\|_{D}\right)^{-1} 1_{\{\boldsymbol{Z}>0\}}\right),
$$

where $Z$ is a generator of the $D$-norm corresponding to the copula expansion of $\left(F_{s}\left(X_{s}\right)\right)_{s \in S}$. Hence, the probability that there is a champion among $\boldsymbol{X}^{(1)}, \ldots, \boldsymbol{X}^{(n)}$ does not depend on the univariate margins, but rather on the copula process of $\boldsymbol{X}$. 
The above remark shows that we do not have to limit our considerations to copula processes. If, for instance, $\boldsymbol{X}$ is an MSP itself with univariate marginal distributions $G_{s}, s \in S$, then $\boldsymbol{\eta}:=\left(\log \left(G_{s}\left(X_{s}\right)\right)\right)_{s \in S}$ is an SMSP. Applying the max-stability of $\boldsymbol{\eta}$, we obtain

$$
\begin{aligned}
\bar{\pi}_{n}(\boldsymbol{X})=\bar{\pi}_{n}(\boldsymbol{\eta}) & =P\left(\boldsymbol{\eta}>\max _{i=1, \ldots, n-1} \boldsymbol{\eta}^{(i)}\right) \\
& =P\left((n-1) \boldsymbol{\eta}>\boldsymbol{\eta}^{(1)}\right) \\
& =\int_{\bar{C}^{-}(S)} P\left((n-1) f>\boldsymbol{\eta}^{(1)}\right)(P * \boldsymbol{\eta})(\mathrm{d} f) \\
& =\int_{\bar{C}^{-}(S)} \exp \left(-(n-1)\|f\|_{D}\right)(P * \boldsymbol{\eta})(\mathrm{d} f) \\
& =E\left(\exp \left(-(n-1)\|\boldsymbol{\eta}\|_{D}\right)\right),
\end{aligned}
$$

where $\|\cdot\|_{D}$ is the $D$-norm corresponding to $\boldsymbol{\eta}$, and $\boldsymbol{\eta}^{(1)}, \boldsymbol{\eta}^{(2)}, \ldots$ are iid copies of $\eta$.

Having established the functional extremal concurrence probability, we can now derive the limit survival function of a complete record. We will have to restrict to the case where $P(\boldsymbol{Z}>0)>0$, which is equivalent to the fact that the extremal concurrence probability is positive, cf. (12).

Just like before, we consider the copula process case first.

Proposition 3.7. In addition to the assumptions of Theorem 3.1, suppose that the generator fulfills $P(\boldsymbol{Z}>0)>0$. Then, for $f \in \bar{E}^{-}(S)$,

$$
\begin{aligned}
P\left(n\left(\boldsymbol{U}^{(n)}-\mathbf{1}\right)>f \mid \boldsymbol{U}^{(n)} \text { is a complete record }\right) \\
=: \bar{H}_{n}(f) \rightarrow_{n \rightarrow \infty} \bar{H}_{D}(f):=\frac{E\left(\Downarrow \max (\boldsymbol{\eta}, f) \Downarrow_{D}\right)}{E\left(\Downarrow \boldsymbol{\eta} \Downarrow_{D}\right)},
\end{aligned}
$$

where $\boldsymbol{\eta}=\left(\eta_{s}\right)_{s \in S}$ is an SMSP with corresponding D-norm $\|\cdot\|_{D}$.

Note that we avoid division by zero in the preceding formula since we assume $P(\boldsymbol{Z}>0)>0$. 
Proof of Proposition 3.7. For the ease of notation, we write $\pi_{n}$ instead of $\bar{\pi}_{n}(\boldsymbol{U})$. We have

$$
\bar{H}_{n}(f)=\frac{\Pi_{n}(f)}{\pi_{n}}:=\frac{P\left(n(\boldsymbol{U}-1)>f, \boldsymbol{U}>\max _{i=1, \ldots, n-1} \boldsymbol{U}^{(i)}\right)}{P\left(\boldsymbol{U}>\max _{i=1, \ldots, n-1} \boldsymbol{U}^{(i)}\right)} .
$$

By Theorem 3.1, it remains to show that for each $f \in \bar{E}^{-}(S)$

$$
n \Pi_{n}(f)=n P\left(n(\boldsymbol{U}-1)>\max \left(f, \boldsymbol{M}^{(n)}\right)\right) \rightarrow_{n \rightarrow \infty} E\left(\gtrless \max (\boldsymbol{\eta}, f) \gtrless_{D}\right),
$$

where $\boldsymbol{M}^{(n)}:=n \max _{i=1, \ldots, n-1}\left(\boldsymbol{U}^{(i)}-1\right)$. This can be done by repeating the arguments of the proof of Theorem 3.1.

Note that another representation of $\bar{H}_{D}(f)$ is given by

$$
\bar{H}_{D}(f)=1-\frac{E\left(\left(\|1 / \boldsymbol{Z}\|_{D}\right)^{-1} \exp \left(\|1 / \boldsymbol{Z}\|_{D} \sup _{s \in S}\left(f(s) Z_{s}\right)\right) \cdot 1_{\{\boldsymbol{Z}>0\}}\right)}{E\left(\left(\|1 / \boldsymbol{Z}\|_{D}\right)^{-1} \cdot 1_{\{\boldsymbol{Z}>0\}}\right)},
$$

where $\boldsymbol{Z}$ is a generator of $\|\cdot\|_{D}$. This is due to Lemma 3.3 .

EXAMPLE 3.8. For the Marshall-Olkin D-norm

$$
\|\boldsymbol{x}\|_{M_{\gamma}}:=\gamma\|\boldsymbol{x}\|_{\infty}+(1-\gamma)\|\boldsymbol{x}\|_{1}, \quad \boldsymbol{x} \in \mathbb{R}^{d}, \gamma \in(0,1),
$$

we obtain with $\mathbf{1}:=(1, \ldots, 1) \in \mathbb{R}^{d}$

$$
\bar{H}_{\gamma}(\boldsymbol{x})=1-\exp \left(\|\mathbf{1}\|_{M_{\gamma}} \max _{i=1, \ldots, d} x_{i}\right), \quad \boldsymbol{x} \leq 0,
$$

which is the survival function of the max-stable $\operatorname{rv}(\eta, \ldots, \eta) /\|\mathbf{1}\|_{\gamma}$, where $\eta$ is standard negative exponentially distributed and $\|\mathbf{1}\|_{M_{\gamma}}=\gamma+d(1-\gamma)$. Note that this rv has complete dependent and identically distributed univariate margins.

Proof. A generator of the Marshall-Olkin $D$-norm $\|\cdot\|_{M_{\gamma}}$ is given by

$$
\boldsymbol{Z}:=\xi(1, \ldots, 1)+(1-\xi) \boldsymbol{Z}^{*}
$$


where $\xi$ is a rv with $P(\xi=1)=\gamma=1-P(\xi=0)$, and $\xi$ is independent of $\boldsymbol{Z}^{*}$ which is a random permutation of the vector $(d, 0, \ldots, 0)$ with equal probability $1 / d$. Obviously, $P(\boldsymbol{Z}>0, \xi=0)=0$. On the other hand, $\xi=1$ implies $\boldsymbol{Z}=1$. Thus, we obtain by (13) for all $\boldsymbol{x} \leq \mathbf{0}$

$$
\begin{aligned}
\bar{H}_{\gamma}(\boldsymbol{x}) & =1-\frac{E\left(\left(\|1 / \boldsymbol{Z}\|_{M_{\gamma}}\right)^{-1} \exp \left(\|1 / \boldsymbol{Z}\|_{M_{\gamma}} \max _{i=1, \ldots, d}\left(x_{i} Z_{i}\right)\right) \cdot 1_{\{\boldsymbol{Z}>0, \xi=1\}}\right)}{E\left(\left(\|1 / \boldsymbol{Z}\|_{M_{\gamma}}\right)^{-1} \cdot 1_{\{\boldsymbol{Z}>0, \xi=1\}}\right)} \\
& =1-\exp \left(\|\mathbf{1}\|_{\left.M_{\gamma} \max _{i=1, \ldots, d} x_{i}\right) .}\right.
\end{aligned}
$$

In order to generalize Proposition 3.7 to stochastic processes in $C(S)$ with arbitrary margins, the following lemma is needed.

Lemma 3.9. Let $f_{n}, n \in \mathbb{N}$, be a sequence of functions in $\bar{E}^{-}(S)$ converging uniformly to $f \in \bar{E}^{-}(S)$. Then, under the conditions and notation of Proposition 3.7,

$$
\bar{H}_{n}\left(f_{n}\right)=\frac{\Pi_{n}\left(f_{n}\right)}{\pi_{n}} \rightarrow_{n \rightarrow \infty} \bar{H}_{D}(f) .
$$

Proof. Let $\varepsilon>0$. Due to the uniform convergence of $f_{n}$, there exists $N \in \mathbb{N}$ such that $f-\varepsilon \leq f_{n} \leq f+\varepsilon$ for $n \geq N$. Assume without loss of generality $f+\varepsilon<0$, otherwise consider $\min (f+\varepsilon, 0)$. Clearly, for such $n$,

$$
\Pi_{n}(f+\varepsilon) \leq \Pi_{n}\left(f_{n}\right) \leq \Pi_{n}(f-\varepsilon) .
$$

Now with $n \rightarrow \infty$, Proposition 3.7 shows

$$
E\left(\inf _{s \in S}\left|\max \left(\eta_{s}, f(s)-\varepsilon\right)\right| Z_{s}\right) \leq \lim _{n \rightarrow \infty} \Pi_{n}\left(f_{n}\right) \leq E\left(\inf _{s \in S}\left|\max \left(\eta_{s}, f(s)+\varepsilon\right)\right| Z_{s}\right) .
$$

Now check

$$
\inf _{s \in S}\left|\max \left(\eta_{s}, f(s) \pm \varepsilon\right)\right| Z_{s} \leq-\eta_{s_{0}} Z_{s_{0}}, \quad s_{0} \in S,
$$

and let $\varepsilon \downarrow 0$. The assertion now follows from the dominated convergence theorem. 
We are now ready to generalize Proposition 3.7 to stochastic processes in $C(S)$ with arbitrary univariate margins. Let $\boldsymbol{X}=\left(X_{s}\right)_{s \in S}$ be a process in $C(S)$ whose univariate marginal dfs $F_{s}(x)=P\left(X_{s} \leq x\right), x \in \mathbb{R}, s \in S$, are continuous and strictly monotonically increasing on their support. Let $\boldsymbol{\vartheta}$ be an MSP with univariate marginal dfs $G_{s}(x)=P\left(\vartheta_{s} \leq x\right), x \in \mathbb{R}, s \in S$. We conclude from de Haan and Lin (2001, Theorem 2.8) that $\boldsymbol{X}$ is in the max-domain of attraction of $\boldsymbol{\vartheta}$ (in the sense of (2)) if and only if the copula process corresponding to $\boldsymbol{X}$, namely

$$
\boldsymbol{U}=\left(U_{s}\right)_{s \in S}:=\left(F_{s}\left(X_{s}\right)\right)_{s \in S}
$$

is in the max-domain of attraction of the SMSP $\boldsymbol{\eta}=\left(\eta_{s}\right)_{s \in S}=:\left(\log \left(G_{s}\left(\vartheta_{s}\right)\right)\right)_{s \in S}$ and the univariate margins fulfill

$$
F_{s}\left(c_{n}(s) x+d_{n}(s)\right)^{n} \rightarrow_{n \rightarrow \infty} G_{s}(x), \quad x \in \mathbb{R},
$$

uniformly for $s \in S$ and locally uniformly for $x \in \mathbb{R}$, where $c_{n} \in C^{+}(S), d_{n} \in C(S)$, $n \in \mathbb{N}$, are the norming functions from (2).

Corollary 3.10. Let $\vartheta$ be an $M S P$ with univariate marginal $d f s G_{s}, s \in S$, and $\boldsymbol{X}^{(1)}, \boldsymbol{X}^{(2)}, \ldots$ be independent copies of a process $\boldsymbol{X} \in \mathcal{D}(\boldsymbol{\vartheta})$ in $C(S)$. Let $c_{n} \in$ $C^{+}(S), d_{n} \in C(S), n \in \mathbb{N}$, be the norming functions from (2), and suppose the univariate margins of $\boldsymbol{X}$ satisfy (14). Put

$$
\boldsymbol{U}=\left(U_{s}\right)_{s \in S}:=\left(F_{s}\left(X_{s}\right)\right)_{s \in S}, \quad \boldsymbol{\eta}=\left(\eta_{s}\right)_{s \in S}=:\left(\log \left(G_{s}\left(\vartheta_{s}\right)\right)\right)_{s \in S},
$$

and let $\|\cdot\|_{D}$ be the D-norm of $\boldsymbol{\eta}$. Choose a generator $\boldsymbol{Z}=\left(Z_{s}\right)_{s \in S}$ of $\|\cdot\|_{D}$ and suppose that $P(\boldsymbol{Z}>0)>0$. Then, for $f \in E(S)$ with $\inf _{s \in S} G_{s}(f(s))>0$,

$$
P\left(\frac{\boldsymbol{X}^{(n)}-d_{n}}{c_{n}}>f \mid \boldsymbol{X}^{(n)} \text { is a complete record }\right) \rightarrow_{n \rightarrow \infty} \bar{H}_{D}(\psi(f))
$$

where $\psi(f)(s)=\log \left(G_{s}(f(s))\right), s \in S$. 
Proof. Denote by $\boldsymbol{U}^{(n)}$ the copula process corresponding to $\boldsymbol{X}^{(n)}, n \in \mathbb{N}$. Taking logarithms, (14) becomes

$$
\sup _{s \in S}\left|n\left(F_{s}\left(c_{n}(s) x+d_{n}(s)\right)-1\right)-\log \left(G_{s}(x)\right)\right| \rightarrow_{n \rightarrow \infty} 0 .
$$

It can be shown by elementary arguments that (15) is equivalent to

$$
\begin{aligned}
& \sup _{s \in S}\left|\psi_{n}(f(s))-\psi(f(s))\right|:= \\
& \quad \sup _{s \in S}\left|n\left(F_{s}\left(c_{n}(s) f(s)+d_{n}(s)\right)-1\right)-\log \left(G_{s}(f(s))\right)\right| \rightarrow_{n \rightarrow \infty} 0
\end{aligned}
$$

for each $f \in E(S)$ with $\inf _{s \in S} G_{s}(f(s))>0$. Hence, Lemma 3.9 and the strict monotonicity of $F_{s}$ entail

$$
\begin{aligned}
& n P\left(\frac{\boldsymbol{X}-d_{n}}{c_{n}}>f, \boldsymbol{X}>\max _{i=1, \ldots, n-1} \boldsymbol{X}^{(i)}\right) \\
& =n P\left(n\left(U_{s}-1\right)>\psi_{n}(f(s)), s \in S, \boldsymbol{U}>\max _{i=1, \ldots, n-1} \boldsymbol{U}^{(i)}\right) \\
& \rightarrow_{n \rightarrow \infty} E\left(\Downarrow \max (\boldsymbol{\eta}, \psi(f)) \Downarrow_{D}\right) .
\end{aligned}
$$

\section{Simple RECORDS FOR MUltivariate observations}

Simple record probability. In the preceding section, we have investigated the (normalized) probability of a complete record and in particular, its limit, the extremal concurrence probability. Now we will repeat this procedure, this time for the simple record probability. Unlike in the previous section, where we were actually dealing with the probability of having a champion, normalizing the record probability with the factor $n$ does not yield an interpretation in terms of a probability in the simple record case.

The following result is the equivalent of Theorem 3.1 and Proposition 3.7 in the context of multivariate simple records. Let $\boldsymbol{X}, \boldsymbol{X}_{1}, \boldsymbol{X}_{2}, \ldots$ be i.id. rv in $\mathbb{R}^{d}$ with 
common continuous df $F$. Recall that $\boldsymbol{X}_{n}$ is a simple record, if

$$
\boldsymbol{X}_{n} \not \leq \max _{1 \leq i \leq n-1} \boldsymbol{X}_{i}
$$

and $\underline{\pi}_{n}(\boldsymbol{X})$ denotes the probability of $\boldsymbol{X}_{n}$ being a simple record within the iid sequence $\boldsymbol{X}_{1}, \boldsymbol{X}_{2}, \ldots$

Theorem 4.1. Let $\boldsymbol{U}_{1}, \boldsymbol{U}_{2}, \ldots$ be independent copies of a $r v \boldsymbol{U} \in \mathbb{R}^{d}$ following a copula $C$. Suppose that $C \in \mathcal{D}(G)$ with $G(\boldsymbol{x})=\exp \left(-\|\boldsymbol{x}\|_{D}\right), \boldsymbol{x} \leq \mathbf{0} \in \mathbb{R}^{d}$. Let $\boldsymbol{\eta}$ be a rv with $d f G$. Then

$$
n \underline{\pi}_{n}(\boldsymbol{U}) \rightarrow_{n \rightarrow \infty} E\left(\|\boldsymbol{\eta}\|_{D}\right)
$$

and

$$
\begin{aligned}
& P\left(n\left(\boldsymbol{U}_{n}-\mathbf{1}\right) \leq \boldsymbol{x} \mid \boldsymbol{U}_{n} \text { is a simple record }\right) \\
& \rightarrow_{n \rightarrow \infty} H_{D}(\boldsymbol{x}):=\frac{E\left(\|\min (\boldsymbol{x}, \boldsymbol{\eta})\|_{D}\right)-\|\boldsymbol{x}\|_{D}}{E\left(\|\boldsymbol{\eta}\|_{D}\right)}, \quad \boldsymbol{x} \leq \mathbf{0} \in \mathbb{R}^{d} .
\end{aligned}
$$

In the one dimensional case $d=1$ we obtain $H_{D}(x)=\exp (x), x \leq 0$. Note, however, that $H_{D}$ is not a probability df in general. Take, for instance, $\|\cdot\|_{D}=$ $\|\cdot\|_{1}$, which is the largest $D$-norm. In this case the components $\eta_{1}, \ldots, \eta_{d}$ of $\boldsymbol{\eta}$ are independent and we obtain for $\boldsymbol{x}=\left(x_{1}, \ldots, x_{d}\right) \leq \mathbf{0} \in \mathbb{R}^{d}$

$$
H_{1}(\boldsymbol{x})=\frac{\sum_{i=1}^{d}\left(E\left(\left|\min \left(x_{i}, \eta_{i}\right)\right|\right)-\left|x_{i}\right|\right)}{\sum_{i=1}^{d} E\left(\left|\eta_{i}\right|\right)}=\frac{\sum_{i=1}^{d} \exp \left(x_{i}\right)}{d} .
$$

This is not a probability df on $(-\infty, 0]^{d}$ as, for example, $H_{1}(\boldsymbol{x})$ does not converge to zero if only one component $x_{i}$ converges to $-\infty$. Even more, choose $\boldsymbol{a} \leq \boldsymbol{b} \leq \mathbf{0} \in \mathbb{R}^{d}$. If $H_{1}$ would define a probability measure $Q$ on $(-\infty, 0]^{d}$, then the probability $Q([\boldsymbol{a}, \boldsymbol{b}])$ were given by

$$
\Delta_{\boldsymbol{a}}^{\boldsymbol{b}} H_{1}=\sum_{\boldsymbol{m} \in\{0,1\}^{m}}(-1)^{d-\sum_{1 \leq j \leq d} m_{j}} H_{1}\left(b_{1}^{m_{1}} a_{1}^{1-m_{1}}, \ldots, b_{d}^{m_{d}} a_{d}^{1-m_{d}}\right)
$$


But elementary computations show that $\Delta_{\boldsymbol{a}}^{b} H_{1}=0$, i.e., $Q$ is the null measure on $(-\infty, 0]$.

Instead one can define $Q$ on $[-\infty, 0]^{d} \backslash\{-\infty\}$ by putting for $x_{i} \leq 0$ and $i=$ $1, \ldots, d$

$$
Q\left(\{-\infty\} \times \cdots \times\{-\infty\} \times\left(-\infty, x_{i}\right] \times\{-\infty\} \times \cdots \times\{-\infty\}\right):=\frac{\exp \left(x_{i}\right)}{d}
$$

Then $Q$ has its complete mass on the set $\left\{\bigcup_{i=1}^{d}\left(\{-\infty\}^{i-1} \times(-\infty, 0] \times\{-\infty\}^{d-i}\right)\right\}$ and

$$
\begin{aligned}
& Q\left(\times_{i=1}^{d}\left[-\infty, x_{i}\right] \backslash\{-\infty\}\right) \\
& =Q\left(\bigcup_{i=1}^{d}\left(\{-\infty\} \times \cdots \times\{-\infty\} \times\left(-\infty, x_{i}\right] \times\{-\infty\} \times \cdots \times\{-\infty\}\right)\right) \\
& =\sum_{i=1}^{d} Q\left(\{-\infty\} \times \cdots \times\{-\infty\} \times\left(-\infty, x_{i}\right] \times\{-\infty\} \times \cdots \times\{-\infty\}\right) \\
& =\frac{1}{d} \sum_{i=1}^{d} \exp \left(x_{i}\right) .
\end{aligned}
$$

This approach is closely related to the formulation of the exponent measure theorem as in Balkema and Resnick (1977) and Vatan (1985).

Take, on the other hand, $\|\cdot\|_{D}=\|\cdot\|_{\infty}$, which is the least $D$-norm. In this case, the components $\eta_{1}, \ldots, \eta_{d}$ of $\boldsymbol{\eta}$ are completely dependent, i.e., $\eta_{1}=\eta_{2}=\cdots=\eta_{d}$ a.s. and, thus,

$$
\begin{aligned}
H_{\infty}(\boldsymbol{x}) & =E\left(\left\|\left(\min \left(x_{i}, \eta_{1}\right)\right)_{i=1}^{d}\right\|_{\infty}\right)-\|\boldsymbol{x}\|_{\infty} \\
& =E\left(\max \left(\|\boldsymbol{x}\|_{\infty}, \eta_{1}\right)\right)-\|\boldsymbol{x}\|_{\infty} \\
& =\exp \left(-\|\boldsymbol{x}\|_{\infty}\right), \quad \boldsymbol{x}=\left(x_{1}, \ldots, x_{d}\right) \leq \mathbf{0} \in \mathbb{R}^{d}
\end{aligned}
$$

which is a max-stable distribution (MSD). 
Proof of Theorem 4.1. Let $\boldsymbol{Z}$ be a generator of $\|\cdot\|_{D}$, independent of $\boldsymbol{\eta}$. Theorem 3.1 , the inclusion-exclusion principle and (8) yield

$$
\begin{aligned}
n \underline{\pi}_{n}(\boldsymbol{U}) & =n P\left(\boldsymbol{U} \not \max _{i=1, \ldots, n-1} \boldsymbol{U}_{i}\right) \\
& =n P\left(\bigcup_{j=1}^{d}\left\{U_{j}>\max _{i=1, \ldots, n-1} U_{i, j}\right\}\right) \\
& =\sum_{\emptyset \neq T \subset\{1, \ldots, d\}}(-1)^{|T|-1} n P\left(U_{j}>\max _{i=1, \ldots, n-1} U_{i, j}, j \in T\right) \\
& \rightarrow n \rightarrow \infty \sum_{\emptyset \neq T \subset\{1, \ldots, d\}}(-1)^{|T|-1} E\left(\min _{j \in T}\left|\eta_{j}\right| Z_{j}\right) \\
& =E\left(\max _{j=1, \ldots, d}\left|\eta_{j}\right| Z_{j}\right) \\
& =E\left(\|\boldsymbol{\eta}\|_{D}\right) .
\end{aligned}
$$

Similarily, one can use Proposition 3.7 in order to show for $\boldsymbol{x} \leq \mathbf{0} \in \mathbb{R}^{d}$

$$
n P\left(n(\boldsymbol{U}-1) \not \leq \min \left(\boldsymbol{x}, \boldsymbol{M}_{n}\right)\right) \rightarrow_{n \rightarrow \infty} E\left(\|\min (\boldsymbol{x}, \boldsymbol{\eta})\|_{D}\right),
$$

where $\boldsymbol{M}_{n}:=n \max _{i=1, \ldots, n-1}\left(\boldsymbol{U}_{n}-1\right) \rightarrow_{\mathcal{D}} \boldsymbol{\eta}$. In summary, taking into account (6), we obtain

$$
\begin{aligned}
n P(\boldsymbol{U} & \left.\leq 1+\frac{\boldsymbol{x}}{n}, \boldsymbol{U} \not \max _{i=1, \ldots, n-1} \boldsymbol{U}_{i}\right) \\
= & n P\left(n(\boldsymbol{U}-1) \not \leq \min \left(\boldsymbol{x}, \boldsymbol{M}_{n}\right)\right)-n P\left(\boldsymbol{U} \not \leq 1+\frac{\boldsymbol{x}}{n}\right) \\
& \rightarrow_{n \rightarrow \infty} E\left(\|\min (\boldsymbol{x}, \boldsymbol{\eta})\|_{D}\right)-\|\boldsymbol{x}\|_{D} .
\end{aligned}
$$

The arguments in the preceding proof can easily be repeated to extend Theorem 4.1 to the case of a general $\mathrm{rv} \boldsymbol{X} \in \mathbb{R}^{d}$, whose $\mathrm{df}$ is in the domain of attraction of an MSD. Denote by

$$
C_{F}(\boldsymbol{u}):=F\left(F_{1}^{-1}\left(u_{1}\right), \ldots, F_{d}^{-1}\left(u_{d}\right)\right), \quad \boldsymbol{u}=\left(u_{1}, \ldots, u_{d}\right) \in[0,1]^{d},
$$


the copula of a continuous df $F$ on $\mathbb{R}^{d}$, where $F_{i}$ is the $i$-th univariate marginal df and $F_{i}^{-1}$ its quantile function.

Corollary 4.2. Let $\boldsymbol{X}_{1}, \boldsymbol{X}_{2}, \ldots$ be independent copies of a rv $\boldsymbol{X} \in \mathbb{R}^{d}$, whose $d f$ $F$ is continuous and its copula $C_{F}$ satisfies $C_{F} \in \mathcal{D}(G), G(\boldsymbol{x})=\exp \left(-\|\boldsymbol{x}\|_{D}\right)$, $\boldsymbol{x} \leq \mathbf{0} \in \mathbb{R}^{d}$. We require in addition that each univariate margin $F_{i}$ of $F$ is in the domain of attraction of a univariate $M S D G_{i}$, i.e., there are constants $a_{n i}>0$, $b_{n i} \in \mathbb{R}, n \in \mathbb{N}$, such that for $i=1, \ldots, d$

$$
n\left(1-F\left(a_{n i} x+b_{n i}\right)\right) \rightarrow_{n \rightarrow \infty}-\log \left(G_{i}(x)\right)=:-\psi_{i}(x), \quad x \in \mathbb{R}: G_{i}(x)>0 .
$$

Then we obtain with $\boldsymbol{a}_{n}:=\left(a_{n 1}, \ldots, a_{n d}\right), \boldsymbol{b}_{n}:=\left(b_{n 1}, \ldots, b_{n d}\right)$ and $\boldsymbol{\psi}(\boldsymbol{x}):=$ $\left(\psi_{1}\left(x_{1}\right), \ldots, \psi_{d}\left(x_{d}\right)\right), \boldsymbol{x}=\left(x_{1}, \ldots, x_{d}\right), G_{i}\left(x_{i}\right)>0, i=1, \ldots, d:$

$$
P\left(\frac{\boldsymbol{X}_{n}-\boldsymbol{b}_{n}}{\boldsymbol{a}_{n}} \leq \boldsymbol{x} \mid \boldsymbol{X}_{n} \text { is a simple record }\right) \rightarrow_{n \rightarrow \infty} H_{D}(\boldsymbol{\psi}(\boldsymbol{x})) .
$$

Note that in the case $d=1$

$$
H_{D}(\psi(x))=\exp (\psi(x))=G(x), \quad G(x)>0 .
$$

Note, moreover, that the assumptions on the df $F$ in the preceding theorem are equivalent with the condition $F \in \mathcal{D}(G)$, where $G$ is a $d$-dimensional MSD, together with the condition that $F$ is continuous.

Proof of Corollary 4.2. Assume the representation

$$
\boldsymbol{X}=\left(F_{1}^{-1}\left(U_{1}\right), \ldots, F_{d}^{-1}\left(U_{d}\right)\right)=: \boldsymbol{F}^{-1}(\boldsymbol{U}),
$$

where $\boldsymbol{U}=\left(U_{1}, \ldots, U_{d}\right)$ follows the copula $C$ of $\boldsymbol{X}$. Repeating the arguments in the proof of Theorem 4.1 now implies the assertion.

In Corollary 3.2, we have investigated the expected number of complete records as the sample size goes to infinity, which can be done for simple records analogously. 


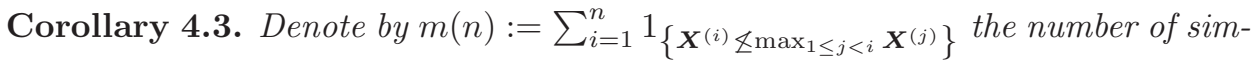
ple records among $\boldsymbol{X}^{(1)}, \ldots, \boldsymbol{X}^{(n)}$. Then

$$
\frac{E(m(n))}{\log (n)} \rightarrow_{n \rightarrow \infty} E\left(\|\boldsymbol{\eta}\|_{D}\right)
$$

The next example shows some connection between the Fréchet and the Weibull model, and provides in particular closed formulas for $E\left(\Downarrow \boldsymbol{\eta} \mathbb{\lambda}_{\lambda}\right)$ and $E\left(\|\boldsymbol{\eta}\|_{\lambda}\right)$.

EXAmple 4.4 (Fréchet model). Choose $\lambda>1$. Let $\boldsymbol{\eta}$ be a max-stable rv in $\mathbb{R}^{d}$ with df $P(\boldsymbol{\eta} \leq \boldsymbol{x})=\exp \left(-\|\boldsymbol{x}\|_{\lambda}\right), \boldsymbol{x} \leq 0$. Let $\boldsymbol{Z}_{F}$ a Fréchet-based generator of $\|\cdot\|_{\lambda}$ (see Example 2.5), and $\boldsymbol{Z}_{W}$ a Weibull-based generator of $\|\cdot\|_{W_{\lambda}}$ (see Example 2.6). We know from Dombry et al. (2015, Example 1) that

$$
E\left(\Downarrow \boldsymbol{\eta} \Downarrow_{\lambda}\right)=\frac{\Gamma(d-1 / \lambda)}{(d-1) ! \Gamma(1-1 / \lambda)}=\prod_{i=1}^{d-1}\left(1-\frac{1}{\lambda i}\right) .
$$

Analogously, one can show

$$
E\left(\left\|\boldsymbol{Z}_{W}\right\|_{\lambda}\right)=\frac{\Gamma(d+1 / \lambda)}{(d-1) ! \Gamma(1+1 / \lambda)}=\prod_{i=1}^{d-1}\left(1+\frac{1}{\lambda i}\right) .
$$

Futhermore, (10) together with Lemma 3.3 yields

$$
E\left(\gtrless \boldsymbol{Z}_{F} \Downarrow_{W_{\lambda}}\right)=E\left(\left\|1 / \boldsymbol{Z}_{F}\right\|_{\lambda}^{-1}\right)=E\left(\Downarrow \boldsymbol{\eta} \Downarrow_{\lambda}\right) .
$$

On the other hand, it is easy to see that $\left(\Gamma(1-1 / \lambda) \Gamma(1+1 / \lambda) \boldsymbol{Z}_{W}\right)^{-1}$ is also a generator of $\|\cdot\|_{\lambda}$, which yields in turn

$$
E\left(\Downarrow \boldsymbol{Z}_{F} \Downarrow_{W_{\lambda}}\right)=E\left(\Downarrow \boldsymbol{Z}_{W} \Downarrow_{\lambda}\right) .
$$

Altogether, we obtain

$$
E\left(\Downarrow \boldsymbol{\eta} \|_{\lambda}\right)=E\left(\Downarrow \boldsymbol{Z}_{W} \Downarrow_{\lambda}\right),
$$

and hence by (8)

$$
E\left(\|\boldsymbol{\eta}\|_{\lambda}\right)=E\left(\left\|\boldsymbol{Z}_{W}\right\|_{\lambda}\right)=\prod_{i=1}^{d-1}\left(1+\frac{1}{\lambda i}\right) .
$$


Simple record times. It is well known that the record times have infinite expectation for a sequence of univariate iid rv with a common continuous df. This is no longer true in the multivariate case. In this section we give a precise characterization.

Let $\boldsymbol{X}_{1}, \boldsymbol{X}_{2}, \ldots$ be i.id. rv in $\mathbb{R}^{d}$ with common continuous df $F$. We denote by $N(n), n \geq 1$, the simple record times, i.e., those subsequent random indices at which a simple record occurs. Precisely, $N(1)=1$, as $\boldsymbol{X}_{1}$ is, clearly, a simple record, and, for $n \geq 2$,

$$
N(n):=\min \left\{j: j>N(n-1), \boldsymbol{X}_{j} \not \leq \max _{1 \leq i \leq N(n-1)} \boldsymbol{X}_{i}\right\} .
$$

As the df $F$ is continuous, the distribution of $N(n)$ does not depend on $F$ and, therefore, we assume in what follows without loss of generality that $F$ is a copula $C$ on $\mathbb{R}^{d}$, i.e., each component of $\boldsymbol{X}_{i}$ is on $(0,1)$ uniformly distributed.

Conditioning on $\boldsymbol{X}_{1}=\boldsymbol{u}$ yields for $j \geq 2$

$$
\begin{aligned}
P(N(2)=j) & =P\left(\boldsymbol{X}_{2} \leq \boldsymbol{X}_{1}, \ldots, \boldsymbol{X}_{j-1} \leq \boldsymbol{X}_{1}, \boldsymbol{X}_{j} \not \leq \boldsymbol{X}_{1}\right) \\
& =\int_{[0,1]^{d}} C(\boldsymbol{u})^{j-2}(1-C(\boldsymbol{u})) C(\mathrm{~d} \boldsymbol{u}) .
\end{aligned}
$$

Solving the geometric series, we get

$$
E(N(2))=\sum_{j=2}^{\infty} j P(N(2)=j)=\int_{[0,1]^{d}} \frac{1}{1-C(\boldsymbol{u})} C(\mathrm{~d} \boldsymbol{u})+1 .
$$

Now we generalize this formula. Choose $n \in \mathbb{N}$. Partitioning the sample space in disjoint events, we obtain for $k_{n} \geq 1$

$$
\begin{aligned}
& P\left(N(n+1)-N(n)=k_{n}\right)= \\
& =\sum_{k_{1}=2}^{\infty} \sum_{k_{2}=1}^{\infty} \cdots \sum_{k_{n-1}=1}^{\infty} P\left(N(n+1)=\sum_{j=1}^{n} k_{j}, N(n)=\sum_{j=1}^{n-1} k_{j}, \ldots, N(2)=k_{1}\right),
\end{aligned}
$$


and further, similar to the calculation above,

$$
\begin{aligned}
& P\left(N(n+1)=\sum_{j=1}^{n} k_{j}, N(n)=\sum_{j=1}^{n-1} k_{j}, \ldots, N(2)=k_{1}\right)= \\
& \int_{\left\{\boldsymbol{u}_{n} \not \cdots \not \boldsymbol{u}_{1}\right\}} C\left(\boldsymbol{u}_{1}\right)^{k_{1}-2} C\left(\boldsymbol{u}_{2}\right)^{k_{2}-1} \cdots C\left(\boldsymbol{u}_{n}\right)^{k_{n}-1}\left(1-C\left(\boldsymbol{u}_{n}\right)\right) C\left(\mathrm{~d} \boldsymbol{u}_{1}\right) \cdots C\left(\mathrm{~d} \boldsymbol{u}_{n}\right) .
\end{aligned}
$$

Hence, solving all the occuring geometric series yields

$$
E(N(n+1)-N(n))=\int_{\left\{\boldsymbol{u}_{n} \not \cdots \not \boldsymbol{u}_{1}\right\}} \prod_{i=1}^{n} \frac{1}{1-C\left(\boldsymbol{u}_{i}\right)} C\left(\mathrm{~d} \boldsymbol{u}_{1}\right) \cdots C\left(\mathrm{~d} \boldsymbol{u}_{n}\right) .
$$

Furthermore, it is easy to see that for all $k \geq 1$

$$
P\left(N(n+1)-N(n)=k \mid \boldsymbol{X}_{N(n)}=\boldsymbol{u}\right)=C(\boldsymbol{u})^{k-1}(1-C(\boldsymbol{u})),
$$

which means that $N(n+1)-N(n) \mid \boldsymbol{X}_{N(n)}=\boldsymbol{u}$ is geometrically distributed with parameter $1-C(\boldsymbol{u})$. We summarize these results.

Lemma 4.5. Let $\boldsymbol{X}_{1}, \boldsymbol{X}_{2}, \ldots$ be iid rv following a copula $C$ on $[0,1]^{d}$, and denote by $N(n)$ the $n$-th simple record time, $n \in \mathbb{N}$.

(i) For every $n \in \mathbb{N}$

$$
N(n+1)-N(n) \mid \boldsymbol{X}_{N(n)}=\boldsymbol{u} \sim \operatorname{Geom}(1-C(\boldsymbol{u})),
$$

where $\operatorname{Geom}(p)$ denotes the geometric distribution with support $\{1,2, \ldots\}$ and parameter $p \in(0,1]$.

(ii) For every $n \in \mathbb{N}$,

$$
E(N(n+1)-N(n))=\int_{\left\{\boldsymbol{u}_{n} \not \cdots \not \boldsymbol{u}_{1}\right\}} \prod_{i=1}^{n} \frac{1}{1-C\left(\boldsymbol{u}_{i}\right)} C\left(\mathrm{~d} \boldsymbol{u}_{1}\right) \cdots C\left(\mathrm{~d} \boldsymbol{u}_{n}\right) .
$$

Suppose now that $d=1$. Then we have $\boldsymbol{u}=u \in[0,1], C(u)=u$ and

$$
E(N(2))=\int_{0}^{1} \frac{1}{1-u} d u+1=\infty
$$

which is well-known (Galambos (1987, Theorem 6.2.1)). 
Suppose next that $d \geq 2$ and that the margins of $C$ are independent, i.e.,

$$
C(\boldsymbol{u})=\prod_{i=1}^{d} u_{i}, \quad \boldsymbol{u}=\left(u_{1}, \ldots, u_{d}\right) \in[0,1]^{d}
$$

Then we obtain

$$
\int_{[0,1]^{d}} \frac{1}{1-C(\boldsymbol{u})} C(\mathrm{~d} \boldsymbol{u})=\int_{0}^{1} \ldots \int_{0}^{1} \frac{1}{1-\prod_{i=1}^{d} u_{i}} \mathrm{~d} u_{1} \ldots \mathrm{d} u_{d}<\infty
$$

by elementary arguments and, thus, $E(N(2))<\infty$. This observation gives rise to the problem of characterizing those copulas $C$ on $[0,1]^{d}$ with $d \geq 2$, such that $E(N(2))$ is finite. Note that $E(N(2))=\infty$ if the components of $C$ are completely dependent.

The next lemma characterizes finiteness of $E(N(2))$.

Lemma 4.6. Let $\boldsymbol{X}=\left(X_{1}, \ldots, X_{d}\right)$ follow a copula $C$ on $\mathbb{R}^{d}$. Then $E(N(2))<\infty$ iff

$$
\int_{1}^{\infty} P\left(X_{i}>1-\frac{1}{t}, 1 \leq i \leq d\right) \mathrm{d} t<\infty
$$

Condition (19) is trivially satisfied in case of independent components $X_{1}, \ldots, X_{d}$ and $d \geq 2$. Below we will see that it is, roughly, in general satisfied, if there are at least two components that are asymptotically independent.

Proof of Lemma 4.6. Any copula $C$ satisfies the Fréchet-Hoeffding bounds, that is, for $\boldsymbol{u}=\left(u_{1}, \ldots, u_{d}\right) \in[0,1]^{d}$

$$
\max \left(1-d+\sum_{i=1}^{d} u_{i}, 0\right) \leq C(\boldsymbol{u}) \leq \min \left(u_{1}, \ldots, u_{d}\right)
$$

Therefore, we obtain due to the upper bound in (20)

$$
\begin{aligned}
E(N(2))-1 & =\int_{[0,1]^{d}} \frac{1}{1-C(\boldsymbol{u})} C(\mathrm{~d} \boldsymbol{u}) \\
& =E\left(\frac{1}{1-C(\boldsymbol{X})}\right) \\
& =\int_{1}^{\infty} P\left(C(\boldsymbol{X})>1-\frac{1}{t}\right) \mathrm{d} t
\end{aligned}
$$




$$
\leq \int_{1}^{\infty} P\left(X_{i}>1-\frac{1}{t}, 1 \leq i \leq d\right) \mathrm{d} t
$$

On the other hand, the lower bound in (20) yields

$$
\begin{aligned}
E(N(2))-1 & \geq \int_{1}^{\infty} P\left(\sum_{i=1}^{d}\left(1-X_{i}\right)<\frac{1}{t}\right) \mathrm{d} t \\
& \geq \int_{1}^{\infty} P\left(1-X_{i}<\frac{1}{d t}, 1 \leq i \leq d\right) \mathrm{d} t \\
& =d \int_{1 / d}^{\infty} P\left(1-X_{i}<\frac{1}{t}, 1 \leq i \leq d\right) \mathrm{d} t
\end{aligned}
$$

Let $C$ be a copula that is in the domain of attraction of a (standard) max-stable df $G$ on $\mathbb{R}^{d}$, i.e.

$$
C^{n}\left(1+\frac{x}{n}\right) \rightarrow_{n \rightarrow \infty} G(x), \quad x \leq 0 .
$$

which we abbreviate by $C \in \mathcal{D}(G)$. Recall that Proposition 2.1 also applies to the multivariate case, see also Remark 2.3.

Proposition 4.7. Suppose that $C \in \mathcal{D}(G)$, where the $D$-norm corresponding to $G$ satisfies $\geqq 1 \Downarrow_{D}>0$. Then $E(N(2))=\infty$.

Proof. Let $\boldsymbol{X}$ be a rv with df $C$. It is well known from real analyis that

$$
\int_{1}^{\infty} P\left(\boldsymbol{X}>1-\frac{1}{t}\right) \mathrm{d} t<\infty \Longleftrightarrow \sum_{n=1}^{\infty} P\left(\boldsymbol{X}>1-\frac{1}{n}\right)<\infty .
$$

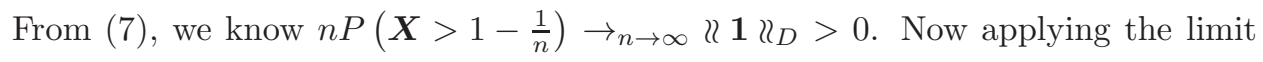
comparison test for an infinite series, we deduce that $\sum_{n=1}^{\infty} P\left(X>1-\frac{1}{n}\right)$ has the same limit behaviour as the harmonic series $\sum_{i=1}^{\infty} \frac{1}{n}$, and hence, $E(N(2))=\infty$ by Lemma 4.6.

Suppose that $C \in \mathcal{D}(G)$. A finite expectation $E(N(2))<\infty$ can, therefore, only occur if $\imath_{1} 1 \gtrless_{D}=0$, which is true, for instance, if $G$ has at least two independent margins. 
Let $\boldsymbol{X}$ follow the $\operatorname{df} C$. Next we show that $E(N(2))$ is typically finite if $\boldsymbol{X}$ has at least two components $X_{j}, X_{k}$ which are tail independent, i. e.

$$
\lim _{u \uparrow 1} P\left(X_{k}>u \mid X_{j}>u\right)=0
$$

In case the limit exists, define the dependence measure

$$
\bar{\chi}:=\lim _{u \uparrow 1} \frac{2 \log (1-u)}{\log \left(P\left(X_{1}>u, X_{2}>u\right)\right)}-1 \in[-1,1],
$$

where $\left(X_{1}, X_{2}\right)$ follows some bivariate copula, cf. Coles et al. (1999); Heffernan (2000). Note that we have $\bar{\chi}=1$ if $X_{1}, X_{2}$ are tail dependent. In the class of (bivariate) copulas that are tail independent, however, $\bar{\chi}$ is a popular measure of tail comparison. For a bivariate normal copula with coefficient of correlation $\rho \in(-1,1)$ it is, for instance, well known that $\bar{\chi}=\rho$.

Proposition 4.8. Let $\boldsymbol{X}=\left(X_{1}, \ldots, X_{d}\right)$ follow a copula $C$ in $\mathbb{R}^{d}$. Suppose that there exist indices $k \neq j$ such that

$$
\bar{\chi}_{k, j}=\lim _{u \uparrow 1} \frac{2 \log (1-u)}{\log \left(P\left(X_{k}>u, X_{j}>u\right)\right)}-1 \in(-1,1) .
$$

Then we have $E(N(2))<\infty$.

Corollary 4.9. We have $E(N(2))<\infty$ for multivariate normal rv unless all components are completely dependent.

Proof of Proposition 4.8. We have

$$
\begin{aligned}
& \int_{1}^{\infty} P\left(X_{i} \geq 1-\frac{1}{t}, 1 \leq i \leq d\right) \mathrm{d} t \\
& \leq \int_{1}^{\infty} P\left(X_{k} \geq 1-\frac{1}{t}, X_{j} \geq 1-\frac{1}{t}\right) \mathrm{d} t \\
& =\int_{1}^{\infty} \exp \left(\frac{\log \left(P\left(X_{k} \geq 1-\frac{1}{t}, X_{j} \geq 1-\frac{1}{t}\right)\right)}{\log \left(\frac{1}{t^{2}}\right)} \log \left(\frac{1}{t^{2}}\right)\right) \mathrm{d} t
\end{aligned}
$$

where

$$
\frac{\log \left(P\left(X_{k} \geq 1-\frac{1}{t}, X_{j} \geq 1-\frac{1}{t}\right)\right)}{\log \left(\frac{1}{t^{2}}\right)} \rightarrow_{t \rightarrow \infty} \frac{1}{1+\bar{\chi}}>\frac{1}{2}
$$


But this implies that the above integral is finite and, thus, the assertion is a consequence of Lemma 4.6.

Next we investigate $E(N(n))$ for $n \geq 2$. As before, let $\boldsymbol{X}_{1}, \boldsymbol{X}_{2}, \cdots$ be an iid sequence of $\mathrm{rv}$ on $\mathbb{R}^{d}$ following a copula $C$. Obviously, $E(N(2))=\infty$ implies $E(N(n))=\infty$ for $n \geq 2$, since $N(n) \geq N(2), n \geq 2$. On the other hand, if $E(N(2))<\infty$, we obtain due to (18) for all $n \geq 2$

$$
\begin{aligned}
E(N(n+1)-N(n)) & =E\left(\frac{1}{1-C\left(\boldsymbol{X}_{1}\right)} \cdots \frac{1}{1-C\left(\boldsymbol{X}_{n}\right)} 1_{\left\{\boldsymbol{X}_{n} \not{z} \cdots \not \boldsymbol{X}_{1}\right\}}\right) \\
& \leq E\left(\frac{1}{1-C\left(\boldsymbol{X}_{1}\right)} \cdots \frac{1}{1-C\left(\boldsymbol{X}_{n}\right)}\right) \\
& \leq\left[E\left(\frac{1}{1-C\left(\boldsymbol{X}_{1}\right)}\right)\right]^{n} \\
& =[E(N(2))-1]^{n},
\end{aligned}
$$

which means that $E(N(n+1)-N(n))<\infty$ as well in that case. Furthermore, we will show below that $E(N(n+1)-N(n))=\infty$ for all $n \in \mathbb{N}$ if $E(N(2))=\infty$. In conclusion, it is sufficient to decide whether $E(N(2))$ is finite or not if expecations of arbitrary simple record times are investigated. We summarize this discussion.

Proposition 4.10. Let $\boldsymbol{X}_{1}, \boldsymbol{X}_{2}, \ldots$ be iid rv following a copula $C$ on $[0,1]^{d}$. Then the following implications hold:

(i) If $E(N(2))=\infty$, then $E(N(n+1)-N(n))=\infty$ for all $n \in \mathbb{N}$.

(ii) If $E(N(2))<\infty$, then $E(N(n+1)-N(n))<\infty$ for all $n \in \mathbb{N}$.

Proof. It remains to proof (ii). We show that $N(n+1)-N(n), n \in \mathbb{N}$, is $\underline{\text { stochastically increasing, }}$ i. e. for every $t \in \mathbb{R}$ and every $n \in \mathbb{N}$

$$
P(N(n+1)-N(n) \leq t) \geq P(N(n+2)-N(n+1) \leq t), \quad t \in \mathbb{R}, n \in \mathbb{N} .
$$

Recall that the df of a $\mathrm{rv} X \sim \operatorname{Geom}(p)$ is given by $P(X \leq t)=1-(1-p)^{\lfloor t\rfloor}$, where $\lfloor t\rfloor=\max \{m \in \mathbb{Z}: m \leq x\}$. Conditioning on $\boldsymbol{X}_{N(n)}=\boldsymbol{x}$, we obtain by 
Lemma 4.5 (i) for each $t \in \mathbb{R}$ and $n \in \mathbb{N}$

$$
\begin{aligned}
& P(N(n+1)-N(n) \leq t) \\
& =\int_{[0,1]^{d}} P\left(N(n+1)-N(n) \leq t \mid \boldsymbol{X}_{N(n)}=\boldsymbol{x}\right) \quad\left(P * \boldsymbol{X}_{N(n)}\right)(\mathrm{d} \boldsymbol{x}) \\
& =\int_{[0,1]^{d}} 1-C(\boldsymbol{x})^{\lfloor t\rfloor}\left(P * \boldsymbol{X}_{N(n)}\right)(\mathrm{d} \boldsymbol{x}) \\
& =1-E\left(C\left(\boldsymbol{X}_{N(n)}\right)^{\lfloor t\rfloor}\right),
\end{aligned}
$$

which shows (21) since $\boldsymbol{X}_{N(n)} \leq \boldsymbol{X}_{N(n+1)}$. Hence,

$$
\begin{aligned}
E(N(n+1)-N(n)) & =\int_{0}^{\infty} P(N(n+1)-N(n)>t \mathrm{~d} t) \\
& \leq \int_{0}^{\infty} P(N(n+2)-N(n+1)>t \mathrm{~d} t) \\
& =E(N(n+2)-N(n+1)) .
\end{aligned}
$$

Computing the distribution of the second record $\boldsymbol{X}_{N(2)}$ is an easy task: Let $\boldsymbol{X}_{1}, \boldsymbol{X}_{2} \ldots$ be independent copies of the $\operatorname{rv} \boldsymbol{X}$ in $\mathbb{R}^{d}$ with $\mathrm{df} F$. We make no further assumption on $F$. Conditioning on $\boldsymbol{X}_{1}=\boldsymbol{y}$ we obtain

$$
\begin{aligned}
P\left(\boldsymbol{X}_{N(2)} \leq \boldsymbol{x}\right) & =\sum_{j=2}^{\infty} P\left(\boldsymbol{X}_{j} \leq \boldsymbol{x}, N(2)=j\right) \\
& =\sum_{j=2}^{\infty} \int_{\mathbb{R}^{d}} P\left(\boldsymbol{X}_{j} \leq \boldsymbol{x}, \boldsymbol{X}_{2} \leq \boldsymbol{y}, \ldots, \boldsymbol{X}_{j-1} \leq \boldsymbol{y}, \boldsymbol{X}_{j} \not \leq \boldsymbol{y}\right) F(\mathrm{~d} \boldsymbol{y}) \\
& =\sum_{j=2}^{\infty} \int_{\mathbb{R}^{d}} F(\boldsymbol{y})^{j-2} P\left(\boldsymbol{X}_{j} \leq \boldsymbol{x}, \boldsymbol{X}_{j} \not \leq \boldsymbol{y}\right) F(\mathrm{~d} \boldsymbol{y}) \\
& =\int_{\mathbb{R}^{d}} P(\boldsymbol{X} \leq \boldsymbol{x}, \boldsymbol{X} \not \leq \boldsymbol{y}) \sum_{j=2}^{\infty} F(\boldsymbol{y})^{j-2} F(\mathrm{~d} \boldsymbol{y}) \\
& =\int_{\mathbb{R}^{d}} \frac{P(\boldsymbol{X} \leq \boldsymbol{x}, \boldsymbol{X} \not \leq \boldsymbol{y})}{1-F(\boldsymbol{y})} F(\mathrm{~d} \boldsymbol{y}) \\
& =\int_{\mathbb{R}^{d}} P(\boldsymbol{X} \leq \boldsymbol{x} \mid \boldsymbol{X} \not \leq \boldsymbol{y}) F(\mathrm{~d} \boldsymbol{y}) .
\end{aligned}
$$


Computation of the distribution of $\boldsymbol{X}_{N(k)}$ for an arbitrary $k \geq 2$ is much more complex, but manageable. Computation of the limit distribution of $\boldsymbol{X}_{N(k)}$, properly linearly standardized, as $k$ tends to infinity, is an open problem. For the univariate case we refer to Galambos (1987, Section 6.4).

\section{REFERENCES}

Arnold, B. C., Balakrishnan, N., and Nagaraja, H. N. (1998). Records. Wiley Series in Probability and Statistics. Wiley, New York.

Aulbach, S., and FAlK, M. (2012a). Local asymptotic normality in $\delta$ neighborhoods of standard generalized Pareto processes. J. Statist. Plann. Inference 142,1339-1347. doi:10.1016/j.jspi.2011.12.011.

Aulbach, S., and Falk, M. (2012b). Testing for a generalized Pareto process. Electron. J. Stat. 6, 1779-1802. doi:10.1214/12-EJS728.

Aulbach, S., Falk, M., and Hofmann, M. (2013). On maxstable processes and the functional D-norm. Extremes 16, 255-283. doi:10.1007/s10687-012-0160-3.

Aulbach, S., Falk, M., Hofmann, M., and Zott, M. (2014). Maxstable processes and the functional D-norm revisited. Extremes 18, 191-212. doi:10.1007/s10687-014-0210-0.

Balkema, A. A., and Resnick, S. I. (1977). Max-infinite divisibility. J. Appl. Probab. 14, 309-319. doi:10.2307/3213001.

Billingsley, P. (1968). Convergence of Probability Measures. Wiley Series in Probability and Mathematical Statistics, 1st ed. Wiley.

Buishand, T. A., de HaAn, L., and Zhou, C. (2008). On spatial extremes: with application to a rainfall problem. Ann. Appl. Stat. 2, 624-642. doi : 10.1214/08-AOAS159.

Coles, S. G., Heffernan, J. E., and Tawn, J. A. (1999). Dependence measure for extreme value analyses. Extremes 2, 339-365. 
Deheuvels, P. (1984). Probabilistic aspects of multivariate extremes. In Statistical Extremes and Applications (J. Tiago de Oliveira, ed.), 117-130. D. Reidel, Dordrecht.

Dombry, C., Ribatet, M., and Stoev, S. (2015). Probabilities of concurrent extremes. Tech. Rep. arXiv:1503.05748 [math.ST].

Falk, M., Hüsler, J., and Reiss, R.-D. (2011). Laws of Small Numbers: Extremes and Rare Events. 3rd ed. Springer, Basel. doi:10.1007/978-3-0348-0009-9.

Ferreira, A., and De HAAn, L. (2014). The generalized Pareto process; with a view towards application and simulation. Bernoulli 20, 1717-1737. doi:10.3150/13-BEJ538.

Galambos, J. (1987). The Asymptotic Theory of Extreme Order Statistics. 2nd ed. Krieger, Malabar.

Giné, E., Hahn, M., and Vatan, P. (1990). Max-infinitely divisible and maxstable sample continuous processes. Probab. Theory Related Fields 87, 139-165. doi:10.1007/BF01198427.

Goldie, C. M., and Resnick, S. I. (1989). Records in a partially ordered set. Ann. Probab. 17, 678-699. doi:10.1214/aop/1176991421.

Goldie, C. M., and Resnick, S. I. (1995). Many multivariate records. Stochastic Process. Appl. 59, 185-216. doi:10.1016/0304-4149(95)00047-B.

de HaAn, L., and Ferreira, A. (2006). Extreme Value Theory: An Introduction. Springer Series in Operations Research and Financial Engineering. Springer, New York. doi:10.1007/0-387-34471-3. See http://people. few.eur.nl/ldehaan/EVTbook.correction.pdf and http://home.isa.utl. pt/ anafh/corrections.pdf for corrections and extensions.

DE HAAn, L., and Lin, T. (2001). On convergence toward an extreme value distribution in $C[0,1]$. Ann. Probab. 29, 467-483. doi:10.1214/aop/1008956340. de Haan, L., audia Neves, C., and Peng, L. (2008). Parametric tail copula estimation and model testing. J. Multivariate Anal. 99, 1260-1275. 
doi:10.1016/j.jmva.2007.08.003.

DE HAAn, L., and RESniCK, S. (1977). Limit theory for multivariate sample extremes. Probab. Theory Related Fields 40,317-337. doi:10.1007/BF00533086.

Heffernan, J. E. (2000). A directory of coefficients of tail dependence. Extremes 3, 279-290.

Hofmann, M. (2012). Contributions to Extreme Value Theory in the Space $C[0,1]$. Ph.D. thesis, University of Würzburg, http://opus.bibliothek. uni-wuerzburg.de/volltexte/2012/7440/.

PiCKAnds, J., III (1981). Multivariate extreme value distributions. Proc. 43th Session ISI (Buenos Aires) 859-878.

Schmidt, R., and Stadtmüller, U. (2006). Non-parametric estimation of tail dependence. Scand. J. Statist. 33, 307-335. doi:10.1111/j.1467-9469.2005.00483.x.

VATAN, P. (1985). Max-infinite divisibility and max-stability in infinite dimensions. In Probability in Banach Spaces V: Proceedings of the International Conference held in Medford, USA, July 1627, 1984 (A. Beck, R. Dudley, M. Hahn, J. Kuelbs, and M. Marcus, eds.), Lecture Notes in Mathematics, vol. 1153, 400425. Springer, Berlin. doi:10.1007/BFb0074963.

Laboratoire de Mathématiques, UfR Sciences et Techniques, Université de FrancheComté, France

E-mail address: clement.dombry@univ-fcomte.fr

Institute of Mathematics, Würzburg, Germany

E-mail address: michael.falk@uni-wuerzburg.de, maximilian.zott@uni-wuerzburg.de 